\title{
A utilization of internal/external quasi-static magnetic field gradients: transport phenomenon and magnetic resonance imaging of solid polymers
}

\begin{abstract}
Naoki Asakawa and Toshiki Obata
Two applications of quasi-static magnetic field gradients in nuclear magnetic resonance spectroscopy are discussed. The first application is conformon transport in the quasi-ordered $\pi$-conjugated polymer, poly(4-methylthiazole-2,5-diyl). With the assistance of proton longitudinal relaxation $\left(R_{1}\right)$ dispersion measurements of the powdered polymer sample, which showed a $\omega^{-1 / 2}$ dependence because of one-dimensional fluctuations in its linear $\pi$-conjugation, one can determine the diffusion coefficient, characteristic transport length, and local magnetic field gradient using proton transverse relaxation $\left(R_{2}\right)$ dispersion measurements under an internal magnetic field gradient and numerical simulations of the spin dynamics. The second application is to demonstrate the possibility for one-dimensional magnetic resonance imaging using an external static magnetic field gradient from a neodymium ferromagnet. High-order primary spin echoes were observed in three-layered thin films consisting of poly(dimethylsiloxane) [PDMS]/poly(tetrafluoroethylene)/PDMS, whereas a comb-like dip in the magnetization amplitude was observed in the quadrature echo spectrum, which means the NMR experiments using the static magnetic field gradient from the ferromagnet were successfully performed. This result could lead to low-cost magnetic resonance imaging instruments for thin films. The possibility of spectral density imaging is also discussed from the viewpoint of variable independence between the static and gradient magnetic fields.
\end{abstract}

Polymer Journal (2012) 44, 855-862; doi:10.1038/pj.2012.101; published online 6 June 2012

Keywords: $\pi$-conjugated polymers; conformon; diffusion; MRI; magnetic field gradient; NMR

\section{INTRODUCTION}

Nuclear magnetic resonance (NMR) spectroscopy under an inhomogeneous magnetic field is a promising, noninvasive method for both the investigation of transport phenomena and magnetic resonance imaging (MRI). Several attempts to collect NMR measurements of ultra-slow diffusion in solids have been recently reported. ${ }^{1-4}$ Among these attempts, the pulse field gradient technique, which uses strong magnetic field gradients, has received a great deal of attention for detecting such diffusion. However, this technique suffers from some critical problems when the target system has a large internal gradient relative to its external and/or internal heterogeneous gradient. The diffusion coefficient of solids is often small, and a local internal gradient, which is, in some cases, heterogeneous and/or anisotropic, can remain, ${ }^{4}$ which is the limiting factor of the method.

The effective gradient of a system with a large internal gradient $\left(G_{\mathrm{i}}\right)$ is not identical to the external one $\left(G_{\mathrm{e}}\right)$, which likely masks the diffusion coefficient. If the root-mean-squared displacement of diffusion were much larger than the periodicity of the local gradient or the characteristic transport length $\left(L_{\mathrm{s}}\right)$, it would not be a problem because the local gradient is expected to average out during diffusion.
By contrast, the local gradient for systems with small diffusion coefficients can remain during the experiments, and the NMR spin echo trains can be further attenuated by the local gradient.

Several approaches to remove the effect of local gradients have been developed so far, such as using an anti-Helmholtz superconducting magnet or the fringe field from a superconducting magnet to produce a large external gradient with the condition $G_{\mathrm{e}} \gg G_{\mathrm{i}}$. With the former technique, a gradient of up to $200 \mathrm{Tm}^{-1}$ can be obtained, ${ }^{2,3}$ while up to $60 \mathrm{Tm}^{-1}$ can be obtained with the latter. ${ }^{1}$ There are also alternative approaches that cancel the local gradient using bipolar gradients $s^{5-8}$ or rotary echos. ${ }^{9}$ By contrast, we pay attention to the local gradient, which is expected to be characteristic of the physical state of the substance, particularly solids, such as the molecular and electronic structure, particle size and shape, and heterogeneity in both density and composition.

In this article, we describe two topics that use quasi-static magnetic field gradients. The first topic is dedicated to measuring the transport properties of the quasi-particles that concern conformational disorder ('conformon') ${ }^{10,11}$ in the head-to-tail regioregular, $\pi$-conjugated polymer poly(4-methylthiazole-2,5-diyl)[P4MeTz] under an internal 
quasi-static magnetic field gradient. The second topic is the proton one-dimensional (1D) MRI (depth-profiling experiment) of threelayered poly(dimethylsiloxane) [PDMS]/poly(tetrafluoroethylene) $[\mathrm{PTFE}] / \mathrm{PDMS}$ thin films under an external static magnetic field gradient from a small neodymium ferromagnet.

\section{Conformon transport measurements using quasi-static internal magnetic field gradients}

For the first topic, we focus on conformon dynamics in the quasiordered phase of P4MeTz. In our previous study, ${ }^{12,13}$ we found that the order-disorder phase transition occurs at $T_{\mathrm{c}}=300 \mathrm{~K}$ for P4MeTz, and the disordered phase dynamically coexists with the ordered phase above $T_{\mathrm{c} .}{ }^{12}$ In general, the elementary excitations and relaxations of $\pi$-conjugated polymers have received a great deal of attention, because they are the basic physical background for various applications such as field-effective transistors, ${ }^{14}$ photovoltaic cells, ${ }^{15}$ and light-emitting diodes. ${ }^{16}$ For instance, the generation and annihilation of excitons, polarons, and bipolarons are typical elementary excitations found in $\pi$-conjugated polymeric systems. 'Conformons' are also elementary excitations that affect the molecular conformation. ${ }^{10,11}$ It is known that some $\pi$-conjugated polymers form a type of mesophase called the quasi-ordered phase defined as a partially disordered crystalline phase. $^{12,17}$ Although one may treat this phase as a nematic liquid crystal with high viscosity, the translational order of the backbone is approximately the same as in the crystalline state. Therefore, the quasi-ordered phase should be distinguished from the nematic phase, where the translational order is reduced by some degree relative to the crystalline state. Furthermore, Yazawa and Asakawa et al. found that the dynamically disordered phase coexists with both the frozen disordered phase and the crystalline phase in the regioregular polymer, poly(3-alkylthiophene-2,5-diyl)s [P3AT], which leads to the argument that the simple expression 'disordered crystal' is inappropriate to describe the complex dynamic phase diagram for P3ATs. ${ }^{18-20}$
Because the quasi-ordered phase in $\pi$-conjugated polymers is highly related to the conjugation length of their $\pi$ electrons, it is important to investigate both the static and dynamic structure of conformons. Despite its importance, information about the dynamics of this phase is still scarce because there are only limited experimental methodologies available to approach the problem. So far, heat capacity, quasielastic neutron scattering, and NMR experiments have been widely performed to explore the collective dynamics of quasiordered structures, such as their charge and spin-density waves, ${ }^{21}$ domain wall dynamics ${ }^{22,23}$ and discommensuration in incommensurate systems. ${ }^{24}$ The dynamics of the quasi-ordered phase of polymers are, however, not yet well established. Here, we examine conformon transport in the quasi-ordered polymer P4MeTz. With the assistance of proton longitudinal relaxation rate $\left(R_{1}\right)$ dispersion measurements of the powdered polymer sample, which showed a $\omega^{-1 / 2}$ dependence because of $1 \mathrm{D}$ fluctuations in its linear $\pi$-conjugation (Figure 1a), one can determine the diffusion coefficient, characteristic transport length, and local magnetic field gradient using proton transverse relaxation rate $\left(R_{2}\right)$ dispersion measurements under an internal magnetic field gradient, which is not possible using only $R_{2}$ dispersion measurements.

\section{MRI using an external static magnetic field gradient}

The second topic concerns conducting MRI under a static external magnetic field gradient. We developed a straightforward MRI method that used the static magnetic field from a small neodymium ferromagnet bar. The maximum magnitude of the field gradient generated by the magnet bar was $100 \mathrm{Tm}^{-1}$, which was larger than normal for a pulsed field gradient, superconducting fringe field or STRAFI. Of course, the sample volume in the current method was less than that required for conventional methods. However, based on most reports related to the micro-imaging of materials, the small sample volumes would not be problematic. In fact, MRI using a field gradient generated by a small magnet bar has a unique trait relative to other methods; that is, one can treat the external magnetic, which is
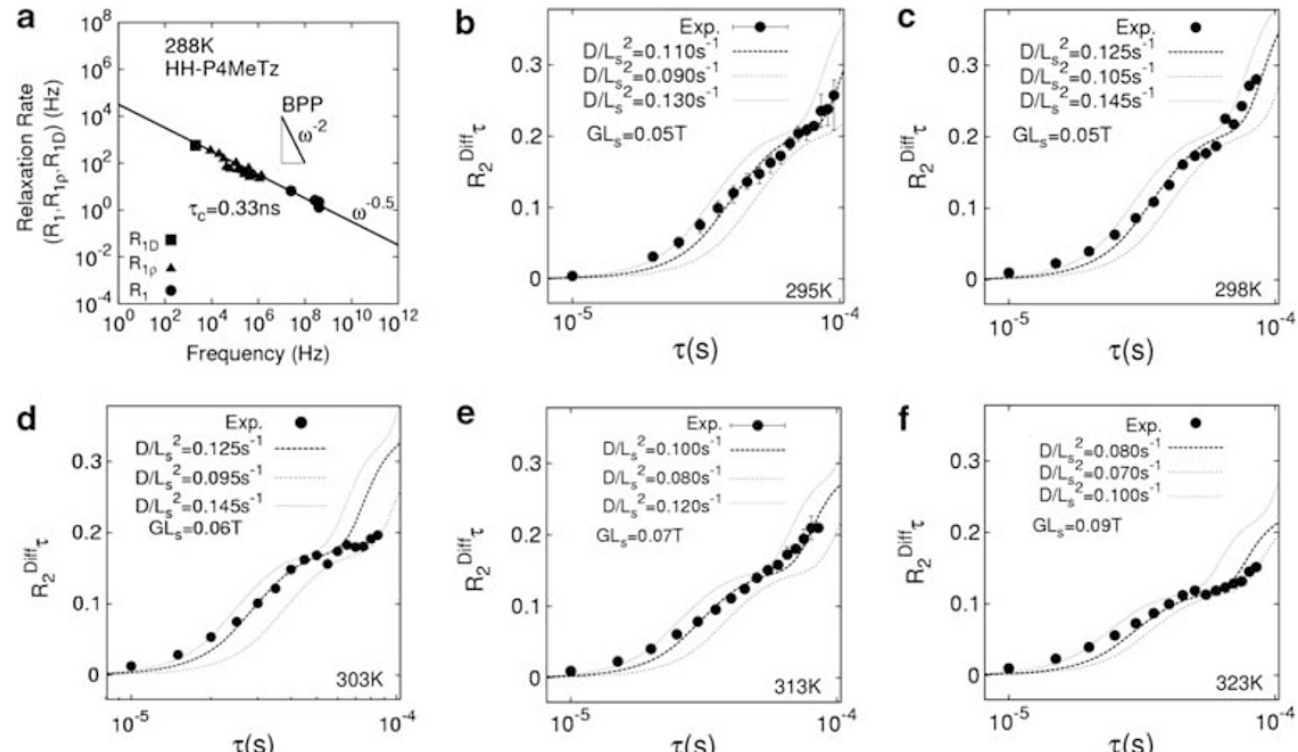

Figure 1 The frequency dependence of the longitudinal proton relaxation rate (a) and the diffusion portion of the transverse proton relaxation exponent vs the spin echo time (b-f). The transverse relaxation rate was measured using the CPMG spin echo. The numerical solution of the $1 \mathrm{D}$ Bloch-Torrey equation was obtained using the Fourier spectrum method. This calculation was performed using the sinusoidal gradient $(f(x)=-0.5 \cos (2 \pi x) ; x:[-1 / 2: 1 / 2])$. The lowest eigen modes for up to 64th iteration were taken into account. The thick dashed line indicates the result of the best fit simulation, while the other lines (thin dashed and dotted lines) are the maximum deviations from the experimental results tolerated for the simulation. 
generated by an electromagnet, and the field gradient as independent variables. What this means is that one can perform variable frequency $R_{1}$ imaging under a constant gradient by adjusting the distance between the ferromagnet and the sample, which allows for MRI experiments to measure the spectral density function of fluctuation. One can explore the local spectral density function, which provides information about the dimensionality of local molecular dynamic fluctuations and the dynamical inhomogeneity often found in solid polymers. In the second half of this article, we present a potential lowcost $1 \mathrm{D}{ }^{1} \mathrm{H}$ MRI method for analyzing solids such as thin polymer films.

\section{EXPERIMENTAL PROCEDURE}

\section{Proton $R_{1}$ and $R_{2}$ dispersion measurements for a $\pi$-conjugated polymer}

The regioregular head-to-head $\mathrm{P} 4 \mathrm{MeTz}\left(M_{\mathrm{w}}=3200\right.$; light scattering $)$ was prepared by the previously reported schemes involving dehalogenative organometallic polycondensation. ${ }^{25}$ For the NMR measurements, we used powder samples of $\mathrm{P} 4 \mathrm{MeTz}$ that were recrystallized by hexafluoroisopropanol.

We carried out variable frequency proton longitudinal relaxation measurements $\left(R_{1}\right.$ dispersion) to investigate the spectral density function of fluctuation of the local field in P4MeTz. The proton longitudinal relaxation times were measured using the following three methods: the saturation-recovery $(\pi / 2-\tau-\pi / 2)$ method $\left(R_{1 \mathrm{H}}\right)$ at the three Larmor frequencies of $25(0.59 \mathrm{~T}), 270(6.34 \mathrm{~T})$, and $400 \mathrm{MHz}(9.4 \mathrm{~T})$; the spin-locking method for the longitudinal relaxation in the rotating frame of a RF field $\left(R_{1 \rho}\right){ }^{26}$ and the Jeener-Broekaert method for the longitudinal relaxation rate of the dipolar order $\left(R_{1 \mathrm{D}}\right) \cdot{ }^{27} R_{1 \rho}$ and $R_{1 \mathrm{D}}$ were measured at a resonance frequency of $400 \mathrm{MHz}$. Commercial NMR spectrometers (Varian, CA, USA; UnityINOVA400 and JEOL, Tokyo, Japan; GSX270) and a home-built low-field NMR spectrometer (for experiments at $25 \mathrm{MHz}$ ) were used. To measure $R_{1 \rho}$ over a wide range of radio-frequency (RF) fields, we used a home-built probe containing a solenoid-type microcoil with an outer radius of $1.2 \mathrm{~mm}$ and a length of $2.5 \mathrm{~mm}$. The intensity of the RF field ranged from 43 to $1.37 \mathrm{MHz}$.

The proton $R_{2}$ dispersion measurements were performed at a resonance frequency of $400 \mathrm{MHz}$. The Carr-Purcell Meiboom-Gill (CPMG) methods were used to observe the diffusion-like motion in the crystal under the local magnetic field gradient. ${ }^{12,28,29}$ The intensity of the RF field was set to $50 \mathrm{kHz}$. These experiments were performed as a function of the echo time. In the absence of diffusion, the CPMG method yields a fixed value for $R_{2}$ independent of the echo time. However, when diffusive motion exists, the value of $R_{2}$ varies with the echo time.

Because the diffusion coefficient of a 1D system can be estimated from the $R_{1}$ dispersion measurements, ${ }^{30,31}$ one can uniquely determine the diffusion coefficient $(D)$, characteristic length $\left(L_{\mathrm{s}}\right)$, and magnetic field gradient $(G)$ by combining such a system with $R_{2}$ dispersion measurements. The analytical procedure is as follows:

(i) Estimate the diffusion coefficient, $D$, from the slope of the $R_{1}$ vs $\omega$ plot ( $R_{1}$ dispersion experiments), and the second moment of resonance, which was signaled using the Van Vleck method, ${ }^{32}$ the longitudinal relaxation rate shows an $\omega^{-0.5}$ dependence.

(ii) Measure the transverse relaxation using CPMG spin echo as an inverse function of the echo time ( $R_{2}$ dispersion experiments), and numerically simulate the Bloch-Torrey equation as solved via the Fourier spectrum method ${ }^{33}$ (see Theoretical section) to obtain the values for $D L_{\mathrm{s}}{ }^{-2}$ and $L_{\mathrm{s}} G$.

(iii) Determine the set of parameters: $D, L_{\mathrm{s}}$, and $G$ from (i) and (ii).

\section{One-dimensional proton MRI for polymer thin films}

For the MRI experiments using a field gradient generated by a ferromagnet bar, we have developed a versatile, low-field NMR/NQR spectrometer based on a PulseBlaster-DDS-III-100 MHz (SpinCore, FL, USA), using NMR probes with a small ferromagnet (Figure 4$)$. We used a neodymium $\left(\mathrm{Nd}_{2} \mathrm{Fe}_{14} \mathrm{~B}\right)$ magnet as a magnetic field gradient source. Figure 4 shows the schematics of the probe head and the electromagnet poles. We used an ex situ detection ${ }^{34}$ of the NMR signal to extend the applicability of our spectrometer to versatile experiments, such as NMR, conducted under an electric field, NMR using unordinary sample shapes and 1D MRI. Three-layered thin films of PDMS (thickness: $200 \mu \mathrm{m}) /$ PTFE $(400 \mu \mathrm{m}) /$ PDMS $(200 \mu \mathrm{m})$, which were purchased from As One, Inc.(Osaka, Japan), were used for the samples. A sample with a surface area of $7 \mathrm{~mm} \times 7 \mathrm{~mm}$ was prepared for the NMR measurements. The proton resonance frequency was set to $27.2 \mathrm{MHz}$ and the spectral width was $2 \mathrm{MHz}$. Because the experiments occur under such a large magnetic field gradient, one cannot apply a homogeneous RF field to the sample and cannot, therefore, accurately determine the RF intensity over the entirety of the spectral width. This intensity was nominal $(50 \mathrm{kHz})$ as determined via an experiment using the same probe but without the neodymium ferromagnet. Two spin echo NMR methods, two pulse Hahn echo (TPHE) ${ }^{35}$ and quadrature echo, ${ }^{36}$ were performed on the film.

These experiments were performed using a home-built NMR spectrometer that can operate at $5-30 \mathrm{MHz}$. The PulseBlaster board produces both rectangular digital and RF pulses. Although the spectrometer is quite primitive and far removed from the state-of-art, most fundamental capabilities, such as versatile pulse programming with a time resolution of $10 \mathrm{~ns}$ (the minimum duration was $80 \mathrm{~ns}$ ), and high-speed digital-analog conversion, are present. This spectrometer can be divided to six sections: the transmitter, receiver, duplexer, probe, magnet, and control sections.

In the transmitter section, a step attenuator (JFW Industries, IN, USA; 50 P077; DC- $1000 \mathrm{MHz}, 0-63 \mathrm{~dB}$ ) was used to control the RF intensity. Before applying the RF signal, a band-pass filter (Mini-circuits, NY, USA; BBP-30) was used to reduce any unwanted noise. A power amplifier (Electronic Navigation Industries, NY, USA; LPI-10) was used to create high-power RF pulses. A duplexer consisting of two boxes containing series crossed diodes, grounded crossed diodes, and a quarter-wavelength coaxial cable was used to electrically separate the transmitter and receiver sections. The number of diodes was determined by the electric power used, and the isolation between the transmitter and receiver sides was determined using a network analyzer (Agilent, CA, USA; 8712ET). A commercial, low-noise NMR amplifier (R\&K, Shizuoka, Japan; N141-305AA) was used as a preamplifier. We used four series of double-balanced mixers (Mini-circuits; ZAD-1) and an additional bandpass filter between the preamplifier and the receiver section to protect the receiver from the transmitted RF pulses. Two-step amplifications (Thamway, Shizuoka, Japan; N141-5059A\&B) followed the preamplification. The output signal from a phase-sensitive detector (Thamway; N113-1066H or N1131066A) was audio-amplified (Thamway; N144-1014A). A digital storage oscilloscope (Agilent; 56420) was used for the analog-digital conversion of the detected phase-sensitive complex number signals. The digitized data were transferred to a personal computer via a general purpose interface bus (GPIB), which was controlled by a GPIB board (National Instruments, TX, USA; NI-GPIB-TNT/PCI).

SpinCore's PulseBlasterDDS board has a D-SUB25 output port for a multichannel, high-speed timing generator to control the various instruments. Because of the D-SUB connector of the PulseBlaster board, it is difficult for researchers to feed high-frequency trigger pulses to various instruments, such as analog-digital conversion, amplifier blanking, and receiver blocking, and impedance matching of the transmission lines from the PulseBlaster to the instruments is a concern. Therefore, we developed an interface board based on latch integrated circuits (74HC541).

We also developed a simple NMR probe with a single RF channel, nonmagnetic trimmer capacitors (Voltronics, MD, USA; NMNT50E $(5-50 \mathrm{pF})$ and NMAT55HVE (1.5-55 pF)) and semi rigid coaxial cables (COAX, Yokohama, Japan; SC-358/50-SC). To reduce the proton background signals, a sample coil (radius: $10 \mathrm{~mm}, 14$ turns) was fabricated from poly(tetrafluoroethylene-cohexafluoropropene) covered copper AWG18 wire, and a probe stator was fabricated from PTFE. A neodymium ferromagnet (Neomag, Chiba, Japan; NCB50 $(50 \times 13.5 \times 5 \mathrm{~mm}))$ was fixed to the probe cover and used to generate a static magnetic field gradient. The distance between the ferromagnet and sample can be varied using stainless steel (SUS316) bolts and nuts. The probes are tuned and matched at $27.2 \mathrm{MHz}$ (Q-factor:200) using the network analyzer.

A four-inch pole electromagnet (Applied Magnetics Laboratory, MD, USA; $4 \mathrm{H} 2-45)$ was used as the homogeneous magnetic field source. An electric 
power source (Walkers LDJ, MA, USA; HS-735-4SS) powered the magnet, and a water-circulating system (Thermo Fisher Scientific, MA, USA; NesLab Merlin $\mathrm{M}-33 / \mathrm{PD}-2$ ) cooled both the magnet and power source.

All of the experiments were controlled using an IBM-AT type personal computer with the Linux OS (GNU Debian-3.0/kernel-2.4.20). The Linux device driver for PulseBlasterDDS was provided by SpinCore Technology, Inc. The driver (nigpib-linux-0.8.6) for the NI-GPIB-TNT device was also provided by National Instruments.

\section{THEORY}

In this section, we will briefly describe the theory for simulating the nuclear spin echo amplitude using a static magnetic field gradient to explore the transport phenomenon that compose the first topic of this article. In general, spin-echo experiments under both external and internal magnetic field gradients are well described by the BlochTorrey equation. The 1D Bloch-Torrey equation is shown in the following:

$$
\frac{\partial M}{\partial t}=\tilde{D} \frac{\partial^{2} M}{\partial \tilde{x}^{2}}-\tilde{\imath} \tilde{\gamma} f(\tilde{x}) M,
$$

Where the tilde denotes dimensionless parameters required for the mathematical problem to be considered general. The effect of longitudinal relaxation $\left(R_{1}\right)$ and intrinsic transverse relaxation $\left(R_{2}\right)$ are neglected in the original Bloch-Torrey equation. However, these effects must be taken into account for practical applications. The dimensionless diffusion coefficient $(\tilde{D})$, dimensionless gyromagnetic ratio $(\tilde{\gamma})$, and dimensionless $1 \mathrm{D}$ coordinate $(\tilde{x}:[-1 / 2: 1 / 2])$ are defined as follows:

$$
\begin{aligned}
& \tilde{D}=\frac{D \tau}{L_{\mathrm{s}}^{2}}, \\
& \tilde{\gamma}=\tau \gamma F L_{\mathrm{s}}, \\
& \tilde{x}=\frac{x}{L_{\mathrm{s}}},
\end{aligned}
$$

Where $L_{\mathrm{s}}, G, \gamma$ and $\tau$ denote the characteristic transport length, magnetic-field gradient, gyromagnetic ratio of the nucleus, and echo time, respectively. The functional form of the $1 \mathrm{D}$ magnetic field gradient is $f(\tilde{x})$.

One can obtain the following equation for the $k$ th Fourier component by using the spatial Fourier transform on equation (1):

$$
\frac{\mathrm{d} M_{\mathrm{k}}(t)}{\mathrm{d} t}=(\mathbf{W}-i \widetilde{\gamma} \mathbf{B}) M_{\mathrm{k}}(t),
$$

Where $\mathbf{W}$ and $\mathbf{B}$ denote the Laplacian and convolution superoperators, respectively, as defined in the literature. ${ }^{33}$

The time-evolution operator for the echo time, $\tau$, can be defined as follows:

$$
U_{+}=e^{(\mathbf{W}-i \gamma \mathbf{B}) \tau} .
$$

For the two-pulse Hahn echo, the evolution operator is expressed as follows:

$$
U_{2 \tau}=\left(U_{+}\right)^{*} U_{+} \cdot
$$

The $n$th echo of CPMG is defined as follows:

$$
\begin{aligned}
& U_{2 n \tau}=U_{2 \tau}^{\bmod (n, 2)} U_{4 \tau}^{\text {floor }(n, 2),} \\
& U_{4 \tau}=U_{+}\left(U_{+}\right)^{*}\left(U_{+}\right)^{*} U_{+} .
\end{aligned}
$$

Therefore, the $k$ th Fourier component of the magnetization at a time of $t=2 n \tau$ can be calculated by the following:

$$
M_{\mathrm{k}}(t)=U_{2 n \tau} M_{\mathrm{k}}(0),
$$

Where $M(0)$ is the magnetization at $t=0$. Finally, one can calculate the magnetization, $M(\mathrm{t})$, from the inverse Fourier transform of $M_{\mathrm{k}}(\mathrm{t})$ and the summation over all components in real space:

$$
M(t)=\sum_{x=-1 / 2}^{1 / 2} F^{-1}\left[M_{\mathrm{k}}(t)\right] .
$$

\section{RESULTS AND DISCUSSION}

One-dimensional transport phenomenon using an internal magnetic field gradient

The longitudinal relaxation of P4MeTz shows an $\omega^{-0.5}$ dependence at $288 \mathrm{~K}$ as shown by our previous study (Figure 1a). ${ }^{12}$ The $R_{1}$ dispersion results can be understood using a dynamic susceptibility theory based on a $1 \mathrm{D}$ random walk model. ${ }^{30}$ It is clear that $1 \mathrm{D}$ diffusive motion exists in $\mathrm{P} 4 \mathrm{MeTz}$ at the frequencies measured. Using the fluctuation-dissipation theorem, the longitudinal relaxation rate $\left(R_{1}\right)$ is expressed as follows:

$$
\begin{aligned}
& R_{1}=M_{2} J(\omega) \\
& J(\omega)=\frac{1}{\sqrt{2}} D_{\|}^{-1 / 2} \omega^{-1 / 2}\left(\text { if } \omega<D_{\|}\right),
\end{aligned}
$$

Where $J(\omega)$ is the spectral density function, $D_{\|}$is the diffusion rate $(3 \mathrm{GHz}$ at $288 \mathrm{~K})$, and $M_{2}$ is the Van Vleck second moment $(2 \mathrm{kHz})$ of the magnetic interactions affecting the relaxation. ${ }^{32}$ Recent photophysical experiments for several $\pi$-conjugated polymers show that the exciton diffusion length should be on order of $1 \mathrm{~nm}$ and localized by the conformational disorder of the polymer chain. ${ }^{37,38}$ Therefore, one can assume that the jumping length of the conformon in the quasi-ordered phase of P4MeTz should similar to the scale of the monomer. For the 1D random walk model at the monomer scale, $D_{\|} c^{2}=2 D$, where $c$ is the lattice parameter of the crystalline unit cell $(c=0.4 \mathrm{~nm})$. Therefore, we obtained $D=2.4 \times 10^{-10} \mathrm{~m}^{2} \mathrm{~s}^{-1}$ at $288 \mathrm{~K}$. Because P4MeTz contains methyl groups as the only proton source and these methyl groups undergo rapid $C_{3 \mathrm{v}}$ rotation at the experimental temperatures, the proton spin diffusion mechanism does not work for even the solid material. A similar result was observed for ${ }^{2} \mathrm{H}-{ }^{2} \mathrm{H}$ spin diffusion in the ${ }^{2} \mathrm{H}$ exchange NMR of $d_{3}$-P4MeTz. ${ }^{12}$ These results indicate $\mathrm{P} 4 \mathrm{MeTz}$ undergoes an uncorrelated methyl rotation in the quasi-ordered phase. On the basis of our previous study, ${ }^{12}$ the $1 \mathrm{D}$ diffusion of the conformon is an Arrhenius-type thermally activated process, ${ }^{12}$ and the diffusion coefficient, $D(\mathrm{~T})$, at different temperatures, $T$, can be estimated using $D(T=288 \mathrm{~K})$ and an activation energy of $14 \mathrm{~kJ} \mathrm{~mol}^{-1}$.

The CPMG relaxation exponent $\left(R_{2}{ }^{\text {diff }} \tau\right)$ can often be explored as a function of the dimensionless diffusion coefficient $(\tilde{D})$ used as a variable to generalize the theoretical estimation of the relaxation exponent. ${ }^{33,39}$ However, CPMG experiments commonly use echo time, $\tau$, as a variable. Fitting the simulated results to the experiment is performed using the following three steps. In general, the total transverse relaxation rate can be expressed as the sum of the intrinsic, $R_{2}$ int, and diffusion, $R_{2}$ diff, relaxation rates in the following equation:

$$
R_{2}=R_{2}^{\text {int }}+R_{2}^{\text {diff }} \text {. }
$$

First, the diffusion portion of relaxation is obtained by subtracting $R_{2}$ by the intrinsic value, $R_{2}{ }^{\text {int }}$. The intrinsic $R_{2}$ was estimated by extrapolating the $R_{2}$ value to $\tau \rightarrow 0$ in the plot of $R_{2}$ vs $\tau$. ${ }^{12}$ Second, because the value of the relaxation exponent at the transition point between the free diffusion (short-time regime) and localization regimes should be unique for a specific system, ${ }^{39}$ the simulated 
relaxation exponent (axis of ordinate; see Figures 1b-f) was fit to the experimental plots by varying the $L_{\mathrm{s}} G$ value, which was directly connected to $\widetilde{\gamma}$ (equation 3 ). Third, the dimensionless diffusion coefficient (axis of abscissa) was scaled to the echo time using the following equation: $\tau=\tilde{D} \cdot L_{\mathrm{s}}^{2} D^{-1}$ (that is, the scaling factor is $\left.L_{s}^{2} D^{-1}\right)$. A sinusoidal gradient, $f(\tilde{x})=-0.5 \cos (2 \pi \tilde{x})$, was used for this simulation. Using the simulated results for gradients with other periodic functional shapes does not severely affect the 1D diffusion relaxation profile. ${ }^{33}$ To obtain the exact solution of the Bloch-Torrey equation, the lowest eigen modes for up to the first 64 iterations of equation (5) were taken into account. From the entirety of these fittings (Figure 1b), we were able to uniquely determine a set of the three parameters $\left(D, L_{\mathrm{s}}\right.$, and $\left.G\right)$ using the $D$ value determined by the $R_{1}$ dispersion $\left(L_{\mathrm{s}}=50 \mu \mathrm{m}\right.$ and $\left.\mathrm{G}=10 \mathrm{Tcm}^{-1}\right)$.

Figure 2 shows the variations in the characteristic transport length scale $\left(L_{\mathrm{s}}\right)$ and local magnetic field gradient $(G)$ with temperature. We obtained an $L_{\mathrm{s}}$ on the order of ten micrometers with large $G$ values (over $10 \mathrm{Tcm}^{-1}$ ). Both $L_{\mathrm{s}}$ and $G$ increase with increasing temperature. The increased $L_{\mathrm{s}}$ values indicate that the mean free path of the conformon was enhanced. In other words, the percolation length is greater in the disordered domain, which is consistent with the increasing fraction of the disordered phase with temperature elevation. It should be noted that the characteristic length scale of the sample obtained during our experiments was approximately three orders larger than that of an extended single polymer chain ( $\sim 13 \mathrm{~nm})$, which implies that intermolecular hopping of the localized conformon occurs in the quasi-ordered phase. However, it would not be surprising that a discrepancy exists between the hopping distance of the random-walk conformon and the characteristic length scale obtained via $R_{2}$ dispersion experiments, because the diffusion behavior observed at long times $\left(t \gg D_{\|}^{-1}\right)$ can reflect collisionlimited ballistic motion for the conformon rather than just a randomwalk at the periodicity of the quasi-ordered phase. In the long time regime, the diffusion coefficient is given by $D=\langle v \lambda\rangle$, where $v$ and $\lambda$ are the group velocity and mean free path of the conformon wave packet, respectively.

As for the value of $G$, although the reason for such a large internal magnetic field gradient remains unknown, the most plausible cause at the characteristic length scale is the particle size of the powder particle of P4MeTz. The particle size of an RR-P4MeTz powder was several tens of micrometers, which is typical for $\pi$-conjugated polymer powders. Therefore, the large internal magnetic field gradient found

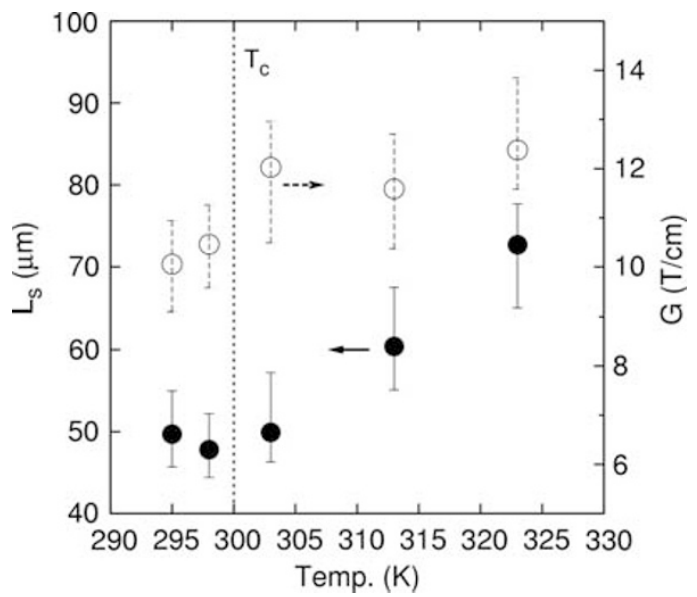

Figure 2 The variation of the characteristic conformon transport length $\left(L_{\mathrm{s}}\right)$ and local magnetic field gradient $(G)$ with temperature. during this work might be caused by the contrast in magnetic susceptibility between the polymer powder particles, which could have large anisotropies in both the molecule and the crystals, or the one-dimensional character of the $\pi$-conjugated polymers.

MRI under an external static magnetic field gradient generated by a small ferromagnet

Figure 3 shows the magnetic field profile for the neodymium magnet. The $y$ value denotes the distance $(\mathrm{mm})$ from the surface of the ferromagnet. At the surface, the magnetic field gradient was estimated as $G=86 \mathrm{~T} / \mathrm{m}$, which is comparable to a typical superconducting magnetic field gradient $(\sim 60 \mathrm{~T} / \mathrm{m})^{1}$ and a GarField magnet for STRAFI measurements $(\sim 20 \mathrm{~T} / \mathrm{m}){ }^{40}$

In our magnetic field set-up, the static homogeneous magnetic field $(H=6.388 \mathrm{kOe})$ from the electromagnet was applied in the direction normal to the axis of easy magnetization of the neodymium ferromagnet (Figure 4). Under these conditions, the rotation angle, $\theta$, of the magnetization of the ferromagnet can be estimated via a wellknown equation: $\theta=\cos ^{-1}\left(-H / H_{\mathrm{k}}\right)$, where $H_{\mathrm{k}}$ is the anisotropy field of the ferromagnet. Because the typical $H_{\mathrm{k}}$ value of a neodymium magnet is $90 \mathrm{kOe}$, the value for $\theta$ was approximately $94^{\circ}$. Therefore, as a first approximation, we neglected the reorientation of the magnetization of the ferromagnet by the magnetic field from the electromagnet.

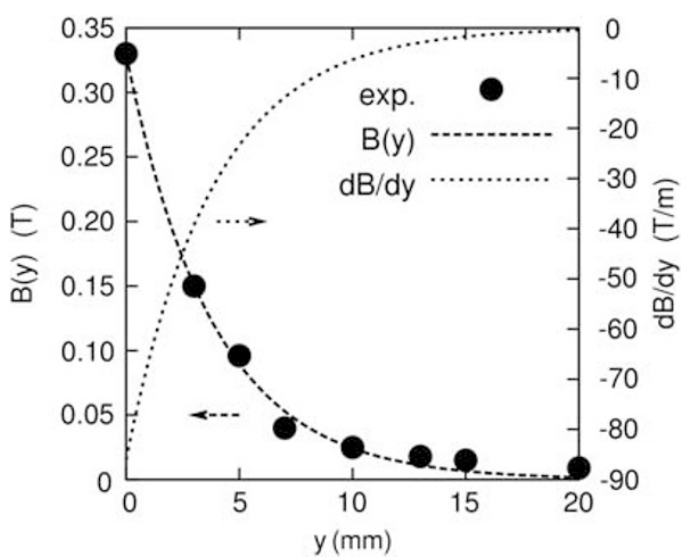

Figure 3 The magnetic field and magnetic field gradient profiles for a small neodymium magnet $\left(\mathrm{Nd}_{2} \mathrm{Fe}_{14} \mathrm{~B}\right)$.

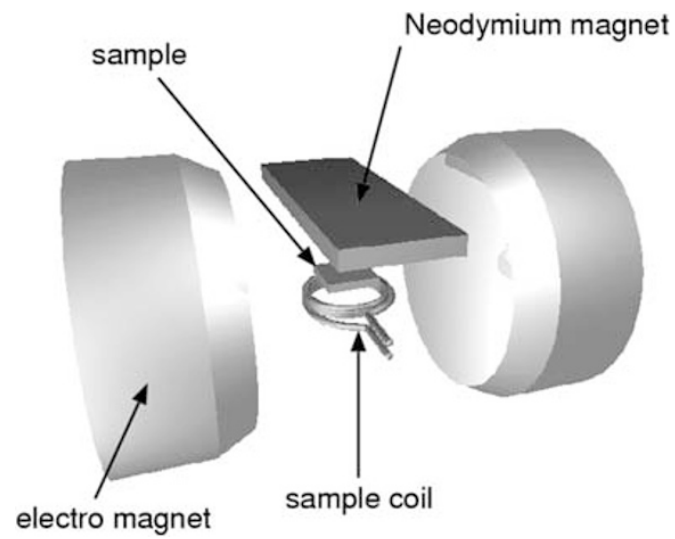

Figure 4 Schematics of the ex situ NMR probe head under the static magnetic field from a small ferromagnet. 


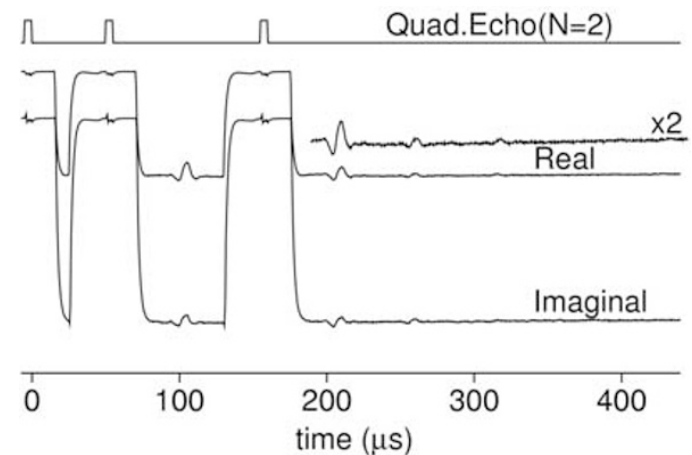

Figure 5 Time course for the $27.2 \mathrm{MHz}$ proton quadrature echo experiment using $N=2$ for a PDMS/PTFE/PDMS three-layered thin films. High-order primary spin echoes were observed in the time range from 25 to $350 \mu \mathrm{s}$. These results indicate that the experiment was performed under a large magnetic field gradient.

To demonstrate our method, the distance from the surface of the neodymium magnet to the three-layered thin films of PDMS/PTFE/ PDMS was set to $10 \mathrm{~mm}$. Figure 5 shows the time-domain signal of the PDMS/PTFE/PDMS thin film measured by the quadrature echo sequence with $N=2$. The large baseline shifted along the vertical axis is due to the receiver blocking pulse. Two high-order primary spin echoes were observed between 250-350 $\mu$ s. According to Bloom, ${ }^{41}$ Randall et al., ${ }^{42}$ and Shakhmuratova et al., ${ }^{43}$ this finding is evidence that the experiment was performed under a large magnetic field gradient. This transient coherence arise from the complex superposition of oscillatory-free induction decays or echoes, which extend further into the time domain because of cumulative dephasing within each pulse of the multiple pulse experiment. ${ }^{43}$

Figure 6 shows a comparison between the 1D MRI spectra obtained using the TPHE and quadrature echo methods for the PDMS/PTFE/ PDMS film. Because PDMS contains methyl protons whereas PTFE is a proton-free material, all of the ${ }^{1} \mathrm{H}$ NMR signals are from PDMS exclusively; that is, the PTFE film simply acts as a spacer that does not contribute any proton magnetization to the spectrum. Therefore, the $1 \mathrm{D}{ }^{1} \mathrm{H}$ image should yield double peaks as shown in Figure 6. It should be noted that the spectrum obtained via the quadrature echo method indicated more dips than that obtained via TPHE. Therefore, we performed numerical simulations using the spin dynamics theory by Benson and $\mathrm{McDonald}^{44}$ to qualitatively understand these oscillatory dips. Figure 7 shows the results of this simulation. Although we assumed a homogeneous nuclear-spin density across the entire spectral range, many dips in the magnetization were visible in the quadrature echo spectrum (Figure $7 \mathrm{~b}$ ). These dips were less pronounced in the TPHE spectrum near the carrier frequency (the center of the spectrum) (Figure 7a). Such behavior was also visible in the experimental spectra shown in Figure 6.

To gain insight into the $1 \mathrm{D}$ images, we performed two convolution processes on the numerical simulations; one blurred the sample shape because of poor sample alignment with respect to the external magnetic field and field gradient, while the other broadened the line to account for $T_{2}^{*}$. Figure $7 \mathrm{~d}$ shows the blurred sample shape after convoluting the original shape (Figure $7 \mathrm{c}$ ) with the sigmoidal function. Because an analytical blurring function can be particularly complicated, it is difficult to know the exact form a priori, and we used a the sigmoidal function $b(v)=[\tanh (A(v+d))-\tanh$ $(A(v-d))] / 2$, where $v$ is frequency, as an approximation. A parameter set $(d, A)=(100 \mathrm{kHz}, 25 \mu \mathrm{s})$ was heuristically determined from these calculations. Figures $7 \mathrm{e}$ and $\mathrm{f}$ show the convoluted power spectra for
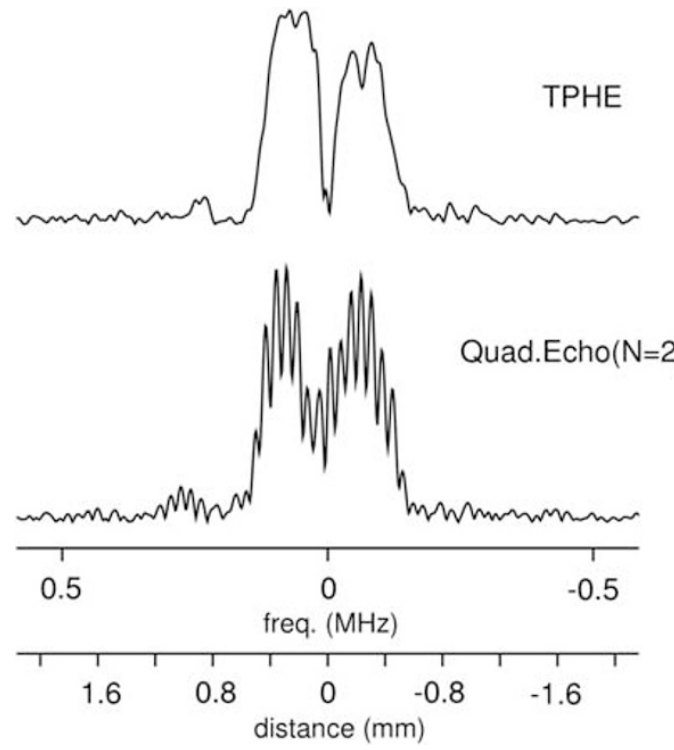

Figure 6 A $27.2 \mathrm{MHz}$ proton one-dimensional magnetic resonance image of the sandwiched PDMS/PTFE/PDMS sample with a comparison between the two-pulse Hahn echo (TPHE) and quadrature echo experiments. Several dips were visible in the MRI using the quadrature echo. The scale in real space is shown in the horizontal axis assuming that the external magnetic field gradient was $6.35 \mathrm{Tm}^{-1}$.

a TPHE homo.

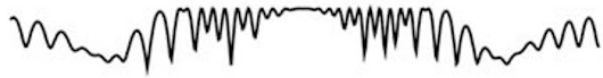

b Quad.Echo homo.

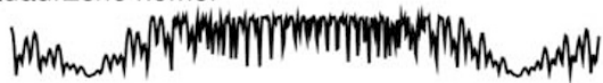

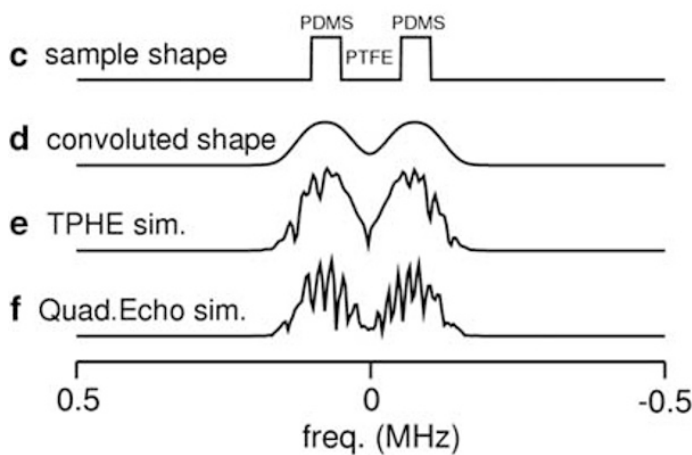

Figure 7 Spectral simulation of the spin echo experiments under a large static magnetic field gradient. The apparent difference between the TPHE and quadrature echo methods can be seen near the center of the spectrum. This simulation was performed based on the spin dynamics theory of Benson and McDonald. ${ }^{44}$ The parameters used in the simulation were $B_{1}=1.174 \mathrm{mT}, \quad G=6.35 \mathrm{Tm}^{-1}$, and $\tau=50 \mu \mathrm{s}$. The TPHE (a) and quadrature echo (b) spectra based on a homogeneous spin density across the entire spectral range, the shape of the three-layered thin PDMS/PTFE/ PDMS film samples (c), the blurred sample shape convoluted using the sigmoidal function (d), and the simulated TPHE (e) and quadrature echo (f) spectra convoluated using the blurred sample shape in (d) are shown. The simulated spectra $(\mathbf{e}, \mathbf{f})$ were also convoluted with the Gaussian function to express the image broadening (see text for the details). 

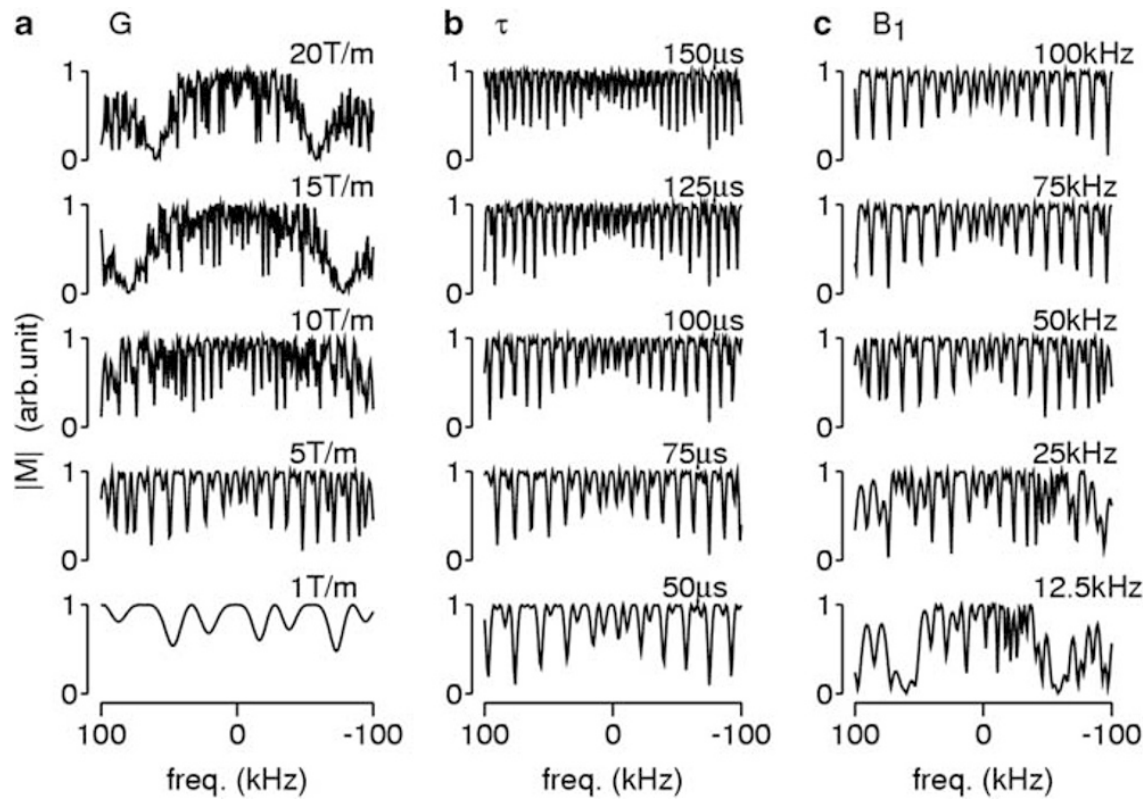

Figure $\mathbf{8}$ The variation in the simulated quadrature echo spectrum with respect to $G(\mathbf{a}), \tau(\mathbf{b})$, and $B_{1}$ (c). For each column in the figure, the two, nonselected parameters $\left(G, \tau\right.$, and $\left.B_{1}\right)$ were fixed to $B_{1}=1.174 \mathrm{mT}(50 \mathrm{kHz}), G=6 \mathrm{~T} / \mathrm{m}$, and $\tau=50 \mu \mathrm{s}$.

the TPHE and quadrature echo spectra, respectively. The second convolution was performed using the Gaussian line shape $g(n)=$ $\exp \left(-\alpha^{2} v^{2}\right)$, where $\alpha=200 \mu \mathrm{s}$.

A numerical simulation of the spin dynamics was performed using $G=6.35 \mathrm{Tm}^{-1}$ as determined by the relationship shown in Figure 3 . The number of dips was roughly reproducible; however, their depth indicated a large discrepancy between the experiment and the numerical simulation. This discrepancy was most likely caused by the complex magnetic field and field gradient maps generated by the neodymium magnet in the static electromagnetic field. Additionally, it may not be practical to ignore the inhomogeneities in $B_{1}$ and $G$. Profiling both the precise magnetic field and gradient with respect to real space remains a subject for future study. Instead, we focused in this article on the qualitative discussion of the dips found in the quadrature echo spectrum. Figure 8 shows the variations in inter-dip frequency with respect to the magnetic field gradient $(G)$, echo time $(\tau)$, and RF intensity $\left(B_{1}\right)$, which indicate they depend greatly on $\tau$ and $G$ but only weakly on $B_{1}$. However, the intensity of $B_{1}$ strongly influences the depth of the dips, most notably at low $B_{1}$ intensities.

Despite the crude relative alignment of the neodymium magnet and sample, we were eventually able to perform one-dimensional MRI with a nominal spatial resolution of several micrometers as shown by the expression of the $200 \mu \mathrm{m}$ PDMS film, using 28 digital points in the best image, which yields $7 \mu \mathrm{m}$ per point. In reality, the $200 \mu \mathrm{m}$ thin film was expressed as 50-60 points, as shown in Figure 6, with blurring approximately doubling the points. Therefore, the practical spatial resolution falls in the order of tens of micrometers and depends on the FWHM of the resonance lines, which are influenced by various factors such as the magnetic susceptibility contrast, intrinsic line width, and so forth.

\section{CONCLUSION}

We investigated the transport property of conformons in the regioregulated $\pi$-conjugated polymer $\mathrm{P} 4 \mathrm{MeTz}$ using combined NMR $R_{1}$ and $R_{2}$ dispersion measurements. For example, there are two possible directions for $1 \mathrm{D}$ diffusion: one along the polymer chain and another along the intermolecular $\pi$-stacking. One plausible scenario would be the $1 \mathrm{D}$ diffusion of the conformon along the polymer chain, because steric hindrance would seem to cause a correlation to the twisting angle of adjacent thiazole rings. However, the mean free path of the conformon is three orders longer than the length of the extended polymer chain, which raises one question: how does the conformon undergo the hopping transport at the end of the chain? We speculate that a rapid hopping transfer, which may be insensitive to NMR, of the conformon between adjacent chains through intermolecular $\pi$-stacking occurs. NMR may only detect the $1 \mathrm{D}$ portion of this anisotropic two-dimensional transport of the conformon. This problem remains an open question.

The method developed in the first half of this article will be generally useful for investigating one-dimensional transport phenomena. Furthermore, recent developments in magnetic resonance force microscopy show that one can perform in situ imaging on a scale of several tens of nanometers using, in general, ultra-high magnetic field gradients of over $10-100 \mathrm{Tcm}^{-1} .45,46$ Until now, there have been limited publications describing spin-echo measurements under such large gradients, which are comparable to the local magnetic field gradient on the atomic scale. ${ }^{47}$ The current method might also be useful to analyze such experiments.

In the second portion, we examined the possibility of MRI using a small neodymium magnet. More precise profiling of the magnetic field and magnetic field gradient around the sample enables us to more precisely perform MRI. The method developed in this research will be applicable to $1 \mathrm{D}$ imaging (depth profiling) of both polymer solids and other heterogeneous thin films. In the future, we will need to develop an NMR theory for systems with variable quantization axes where the effective magnetic field, $B_{\text {eff, }}$ varies in its orientation with respect to the sample.

\section{CONFLICT OF INTEREST}

The authors declare no conflict of interest. 


\section{ACKNOWLEDGEMENTS}

This work was partly supported by the Ministry of Education, Culture, Sports, Science and Technology (Japan) through a Grant-in-Aid for science research (no. 21350125).

1 Kimmich, R., Unrath, W., Schnur, G. \& Rommel, E. NMR measurement of small selfdiffusion coefficients in the fringe field of superconducting magnets. J. Magn. Reson. 91, 136-140 (1991)

2 Chang, I., Fufara, F., Geil, B., Hinze, G., Sillescu, H. \& Tolle, A. New prespectives of $\mathrm{nmr}$ in ultrahigh static magnetic field gradients. J. Non-Crystalline Solids 172-174, 674-681 (1994).

3 Chang, I., Hinze, G., Diezemann, G., Fujara, F. \& Sillescu, H. Self-diffusion coefficient in plastic crystals by multiple-pulse NMR in large static field gradients. Phys. Rev. Lett. 76, 2523-2526 (1996)

4 Ailion, D.C. NMR investigations of ultraslow diffusion in incommensurate insulators and in biomedical systems (e.g. lung). Solid State lonics 125, 251-256 (1999).

5 Karlicek, R.F. \& Lowe, I.J. A modified pulse gradient technique for measuring diffusion in the presence of large background gradients. J.Magn.Reson 37, 75-91 (1980).

6 Cotts, R.M., Hoch, M.J.R., Syn, T. \& Marker, J.T. Pulsed field gradient stimulated echo methods for improved nmr diffusion measurements in heterogeneous systems. J. Magn. Reson. 83, 252-266 (1989).

7 Fordham, E.J., Gibbs, S.J. \& Hall, L.D. Partially restricted diffusion in a permeable sandstone: observations by stimulated Echo PFG NMR. Magn.Reson.Imag 12, 279-284 (1994).

8 Wu, D., Chen, A. \& Johnson, Jr C.S. An improved diffusion-ordered spectroscopy experiment incorporating bipolar-gradient pulses. J. Magn. Reson. A 115, 260-264 (1995)

9 Ardelean, I. \& Kimmich, R. Principles and unconventional aspects of NMR diffusometry. annual repts. NMR Spectroscopy 49, 45-115 (2003).

10 Andrew, E.R. Conformational motion and conformational order-disorder in solids. Phys. Lett.A 34, 30-31 (1971).

11 Volkenstein, M.V. The Conformon. J. Theor. Biol. 34, 193-195 (1972).

12 Mori, S., Inoue, Y., Yamamoto, T. \& Asakawa, N. Dynamics of the quasiordered structure in a $\pi$-conjugated Polymer Poly(4-methylthiazole-2,5-diyl). Phys. Rev. B 71, 054205-1-054205-11 (2005).

13 Mori, S., Yamamoto, T., Asakawa, N., Yazawa, K. \& Inoue, Y. The dynamic structure of regioregulated Poly(4-methylthiazole-2,5-diyl). Polym. J. 40, 475-478 (2008).

14 Tessler, N., Veres, J., Globerman, O., Rappport, N., Preezant, Y., Roichman, Y., Solomesch, O., Tal, S., Gershman, E., Adler, M., Zolotarev, V., Gorelik, V. \& Eichen, Y. In Handbook of Conducting Polymers, 3rd ed.: Processing and Applications (ed. Skotheim, T. A. \& Reynolds, J. R.) Ch. 7 1-42 (CRC Press, 2007).

15 Mozer, A.J. \& Sariciftci, N.S. In Handbook of Conducting Polymers, 3rd ed.: Processing and Applications (ed. Skotheim, T. A. \& Reynolds, J. R.) Ch. 10, 1-37 (CRC Press, 2007).

16 Wessling, B. In Handbook of Conducting Polymers, 3rd ed.: Processing and Applications (ed. Skotheim, T. A. \& Reynolds, J. R.) Ch. 1, 3-75 (CRC Press, 2007).

17 Yang, C., Orfino, F.P. \& Holdcroft, S. A phenomenological model for predicting thermochromism of regioregular and nonregioregular poly(3-alkylthiophenes). Macromolecules 29, 6510-6517 (1996).

18 Yazawa, K., Inoue, Y., Yamamoto, T. \& Asakawa, N. Twist glass transition in regioregulated poly (3-alkylthiophene). Phys. Rev. B 74, 094204-1-094204-12 (2006)

19 Yazawa, K., Inoue, Y., Yamamoto, T. \& Asakawa, N. Dynamic structure of regioregulated poly(alkylthiophene)s. J. Phys. Chem. B 112, 11580-11585 (2008).

20 Yazawa, K., Inoue, Y., Shimizu, T., Tansho, M. \& Asakawa, N. Molecular dynamics of regioregular poly(3-hexylthiophene) Investigated by NMR relaxation and an interpretation of temperature dependent optical absorption. J. Phys. Chem. B 114, 1241-1248 (2010).
21 Mcmillan, W.L. Theory of discommensurations and the commensurate-incommensurate charge-density-wave phase transition. Phys. Rev. B 14, 1496-1502 (1976).

22 Novoselov, K.S., Geim, A.K., Dubonos, S.V., Hill, E.W. \& Grigorieva, I.V. Subatomic movements of a domain wall in the peierls potential. Nature 426, 812-816 (2003).

23 Janssen, T. \& Radulescu, O. Domain wall motion in apriodic crystal systems and magnetoelectrics. Z. Phys. B 104, 657-660 (1997).

24 Blinc, R., Ailion, D.C., Dolinsek, J. \& Zumer, S. Commensurability and defect-induced phason gaps in incommensurate systems. Phys. Rev. Lett. 54, 79-81 (1985).

25 Maruyama, T., Suganuma, H. \& Yamamoto, T. Preparation of $\pi$-conjugated polythiazoles and their electrically conducting properties. Synth. Met. 74, 183-185 (1995)

26 Ailion, D.C. in Advances in Magnetic Resonance (ed. Waugh, J.S.) Ch. 5, 177-227 (Academic, 1971).

27 Jeener, J. \& Broekaert, P. Nuclear magnetic resonance in solids: thermodynamic effects of a pair of RF pulses. Phys. Rev. 157, 232-240 (1967).

$28 \mathrm{Yu}$, I. A method of analyzing restricted diffusion from spin-echo measurements. J. Magn. Reson. A 104, 209-201 (1993).

29 Asakawa, N., Kajikawa, T., Sato, K., Sakurai, M., Inoue, Y. \& Yamamoto, T. Measurements of local magnetic field gradient and diffusion in polymers in the solid state. J. Mol. Struct. 602-603, 455-461 (2002)

30 Nechtschein, M., Devreux, F., Genoud, F., Guglielmi, M. \& Holczer, K. Magneticresonance studies in undoped trans-polyacetylene $(\mathrm{CH})_{x}$ II. Phys. Rev. B 27, 61-78 (1983).

31 McDowell, A.F., Mendelsohn, C.F. \& Conradi, M.S. Two-dimensional diffusion of hydrogen in $\mathrm{ZrBe}_{2} \mathrm{H}_{1.4}$. Phys. Rev. B 51, 6336-6342 (1995).

32 Van Vleck, J.H. The dipolar broadening of magnetic resonance lines in crystals. Phys. Rev. 74, 1168-1183 (1948).

33 Asakawa, N., Matsubara, K. \& Inoue, Y. Low-dimensional lattice diffusion in solids investigated by nuclear spin echo measurements. Chem. Phys. Lett. 406, 215-221 (2005).

34 Meriles, C.A., Sakellariou, D., Heise, H., Moule, A.J. \& Pines, A. Approach to high resolution ex situ NMR spectroscopy. Science 293, 82-85 (2001).

35 Hahn, E.L. Spin echoes. Phys. Rev 80, 580-594 (1950)

36 Powles, J.G. \& Strange, J.H. Zero time resolution nuclear magnetic resonance transient in solids. Proc. Phys. Soc. 82, 6-15 (1963).

37 Hwang, I. \& Scholes, G.D. Electronic energy transfer and quantum-coherence in $\pi$ conjugated polymers. Chem. Mater. 23, 610-620 (2011).

38 Tretiak, S., Saxena, A., Martin, R.L. \& Bishop, A.R. Conformational dynamics of photoexcited conjugated molecules. Phys. Rev. Lett. 89, 097402-1-097402-4 (2002).

39 Axelrod, S. \& Sen, P.N. Nuclear magnetic resonance spin echoes for restricted diffusion in an inhomogeneous field: methods and asymptotic regimes. J. Chem. Phys. 114, 6878-6895 (2001)

40 Glover, P.M., Aptaker, P.S., Bowler, J.R., Ciampi, E. \& McDonald, P.J. A novel highgradient permanent magnet for the profiling of planar films and coatings. J. Magn. Reson. 139, 90-97 (1999).

41 Bloom, S.L. Nuclear induction in inhomogeneous fields. Phys. Rev 98, 1105-111 (1955)

42 Randall, E.W., Samoilenko, A.A. \& Nunes, T. Primary Hahn echoes of high order for solids and liquids. J. Magn. Reson. A116, 259-261 (1995).

43 Shakhmuratova, L.N., Fowler, D.K. \& Chaplin, D.H. Fundamental mechanisms of single-pulse NMR echo formation. Phys. Rev. A 55, 2955-2967 (1997).

44 Benson, T.B. \& McDonald, P.J. The application of spin echoes to stray-field imaging. J. Magn. Reson. B 109, 314-317 (1995).

45 Bruland, K.J., Dougherty, W.M., Garbini, J.L., Sidles, J.A. \& Chao, S.H. Force-detected magnetic resonance in a field gradient of 250000 Tesla per Meter. Appl. Phys. Letts. 73, 3159-3161 (1998).

46 Chao, S.H., Dougherty, W.M., Garbini, J.L. \& Sidles, J.A. Nanometer-scale magnetic resonance imaging. Rev. Sci. Instrum. 75, 1175-1181 (2004).

47 Degen, C.L., lin, Q., Hunkeler, A., Meier, U., Tomaselli, M. \& Meier, B.H. Microscale localized spectroscopy with a magnetic resonance force microscope. Phys. Rev. Lett. 94, 207601-1-207601-4 (2005). 